\title{
Modeling and Characteristic Study of Thin Film Based Biosensor Based on COMSOL
}

\author{
Yang Su \\ Faculty of Information Engineering, Southeast University, Nanjing, Jiangsu 211109, China \\ Correspondence should be addressed to Yang Su; suyang620@163.com
}

Received 9 January 2014; Accepted 6 March 2014; Published 7 April 2014

Academic Editor: Jianguo Du

Copyright (C) 2014 Yang Su. This is an open access article distributed under the Creative Commons Attribution License, which permits unrestricted use, distribution, and reproduction in any medium, provided the original work is properly cited.

\begin{abstract}
The model of thin film based biosensor is built based on COMSOL in order to simulate and optimize the electronic characteristic of dipolar electrodes system and tetrapolar electrodes system. Zones of negative sensitivity exist between current carrying electrodes and voltage measuring electrodes while the polarization occurs at the edges of electrodes. By changing the parameters embracing distance between electrodes, thickness of electrodes, and the width of inner electrodes (for tetrapolar electrodes system only), the polarization and zones of negative sensitivity can be receded to some extent, which improves the system's performance. Tetrapolar electrodes system has less polarization but more negative-sensitivity zones compared to dipolar electrodes system and different setups response differently to these changing parameters.
\end{abstract}

\section{Introduction}

Biological detection techniques exist in multitudes of aspects of human's daily life, especially in the area of chemistry and biology. Numerous devices have already been demonstrated using CMOS technology, such as DNA detection $[1,2]$ and ion sensitive field effect transistor arrays [3]. However, there are postprocessing problems associated with CMOS, for example, circuit passivation [4] and biocompatible electrode deposition, which increase cost and manufacturing complexity. Thin film transistor based devices have the potential to solve some of these issues. For salient instance, they are low cost and use low temperature fabrication processes [5] using glass, flexible plastic, and eventually paper substrates, with no addition processing required. Large TFT arrays have already been demonstrated for flat panel displays so could easily be translated into sensor pixels for massively parallel measurement.

Thin film based biosensor is a kind of cutting-edge technology which can be applied in electronic system for biological sensing. The chip used electrochemical impedance spectroscopy (EIS) as a sensing technique [6-10]. EIS has shown potential to be a sensitive method for label-free sensing. Here, direct electrochemistry of the target analyses is used, with no immobilised enzymes or mediators. At present, bipolar setup with two electrodes which both carry current and measure voltage is widely used. However, this setup can lead to unwanted phenomenon embracing polarization and zones of negative sensitivity [11] which affect the accuracy to some extent. It is essential to design an appropriate thin film sensor in order to reduce the polarization and zones of negative sensitivity as greatly as possible.

This paper concentrates on how three factors embracing geometry, dimension, and configuration for electrodes will affect those two parameters which are mentioned above. At the end of this paper, a better setup of electrodes together with several solutions to improving the performance pertinent to thin-film sensor will be worked out.

\section{Modeling and Experimental Design}

The model of electrodes is based on COMSOL Multiphysics [12] which is a finite element analysis, solver and simulation software package for various physics and engineering applications. In the model, the substrate is made of silica glass while the material of electrodes is gold. The system is studied in $\mathrm{NaCl}$ solution, which is the standard solution for the calibration of electrochemical system. The domain 
frequency is chosen to be $10000 \mathrm{~Hz}$ and the concentration relevant to the solution is $1.76 \mathrm{~S} / \mathrm{m}$ which are bilateral typical values in biosensor test. Three typical types of geometry with regard to electrodes are included in this paper, which are horizontal rectangle, vertical rectangle, and horizontal ring. On the other hand, three factors are taken with reference to dimension, embracing electrode-wise thickness, the distance between electrodes, and the condition where the pair for carrying current and the other for measuring voltage have different size (for tetrapolar electrode system) into consideration, which share affect the polarization and zones of negative sensitivity to some extent. In addition, it is substantial to find out how a tetrapolar electrode system improves the performance compared to the corresponding dipolar one.

In accordance to the simulation results, the strongest polarization appears at the edges of electrodes. As a result, the highest degree is extracted in the case of polarization at edges to access its degree. The sensitivity is a measure of how different component in the measured biomaterial contributes to the total measure impedance, provided that resistivity is uniform throughout the material. The sensitivity $S$ is derived as

$$
S=\frac{J_{1} \cdot J_{2}}{I^{2}}
$$

where $J_{1}$ is the current density measure when a current is injected $I$ between the electrodes for carrying current while $J_{2}$ is the current density measure when the current is injected between the voltage measuring electrodes. A positive value for the sensitivity means that if the resistivity of this volume element is increased, a higher total resistance will be measured. A negative value for the sensitivity, on the other hand, means that increased resistivity in that volume gives a lower total measured resistance, which will lead to errors of measurement. The research on sensitivity is on the basis of the $2 \mathrm{D}$ picture in the light of the distribution of sensitivity. For two-electrode system the sensitivity is always positive in that the two electrodes carry the current and measure the voltage at the same time. For tetrapolar electrode system, the current carrying electrode pair and the voltage measuring pair should be arranged as shown in Figure 1; otherwise sensitivity of most volumes will be negative which is unacceptable [13].

\section{Experimental Tests}

3.1. Electrode Polarization. Polarization is a kind of phenomenon which widely exists in biosensor system. The existence of polarization will lead to additional impedance which will affect the accuracy to some extent. The strongest polarization exists at different position for diversity setups. For dipolar electrode system, the edge of terminal electrode always has the highest degree of polarization while the situation becomes complicated for tetrapolar electrode system. To be more precise, the point polarized mostly of tetrepolar horizontal rectangle electrode system is the edge of inner terminal one while the point changes to the edge of outer terminal electrode when it turns to tetrapolar vertical rectangle one. Additionally, the edge of electrode 1 has the strongest polarization for tetrapolar horizontal ring electrode system. In this paper strongest polarization is called "leading polarization." Virtually, electrode polarization cannot be overcome completely but some measures can be done to minimize it. Three factors of dimension are taken into consideration here, embracing thickness, distance between electrodes, and width of inner electrode pair (for tetrapolar electrode system). According to the simulation, diverse setups of electrode system respond differently to those parameters.

For horizontal rectangle electrode system, the variation of polarization is not regular, which means it is not effective to improve the performance by changing those two factors. It is a bit different when it turns to vertical rectangle electrode system. The degree of leading polarization almost keeps steady as the distance increases while it decreases gradually as the electrodes get thicker. As to horizontal ring electrode system, it is the other way round. In accordance with experiments, it is certain that the increase of distance and thickness make the degree of polarization go down. What is identical in those pictures is that the employment of tetrapolar electrode relieves the effects of polarization greatly. That is to say, using four-electrode system instead of two-electrode system is a brilliant alternative to minimize the effects of polarization.

There are some special results in the condition where the inner electrode pair has a different size from the outer pair. In accordance to Figure 2, the leading polarization pertaining to horizontal rectangle tetrapolar electrode system drops remarkably as the width of inner electrode pair increases. Meanwhile, the leading polarization with regard to vertical rectangle tetrapolar electrode system drops dramatically once the inner pair's height exceeds the outer pair's.

3.2. Zones of Negative Sensitivity and Uniformity of Sensitivity. It is nevertheless a fact that tetrapolar systems are more vulnerable to errors than bipolar system [14]. A fourelectrode system will typically have small volumes with negative sensitivity between current carrying electrodes and voltage measuring ones. As demonstrated in Figure 3, it can be seen explicitly how the zones of negative sensitivity distribute among the space. There are two main negativesensitivity zones for horizontal and vertical rectangle fourelectrode systems while there are four of them. Besides, another factor which is the lowest value of sensitivity is taken into consideration to assess the uniformity of sensitivity. Figure 4 demonstrates how the lowest values relevant to sensitivity of three systems response to the change of distance between electrodes and the width of inner electrode pair. Thickness is not included here in that it nearly has no effect on sensitivity. It can be seen clearly that the lowest values of sensitivity are raised by increasing the distance or the width of inner electrode pair, which means the uniformity is improved to some extent. However, the area of negative-sensitivity zones increases when the distance between electrodes gets larger. The area of negative-sensitive zones mainly depends on the lateral area of current carrying electrode and voltage measuring electrode together with the distance between them. As a result, those two factors can be reduced in order to minimize the volumes of negative sensitivity. On the other 


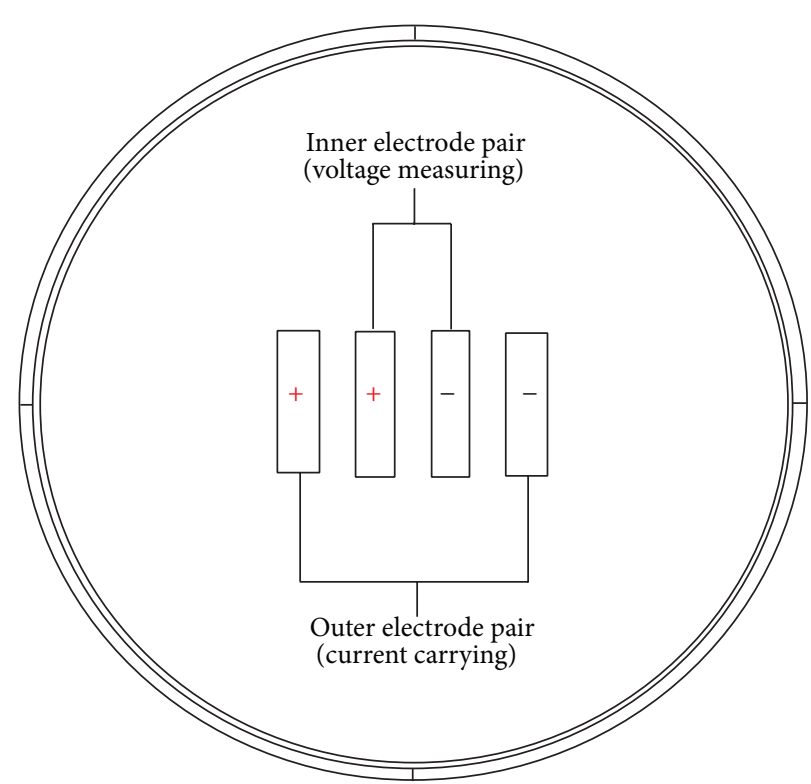

(a)

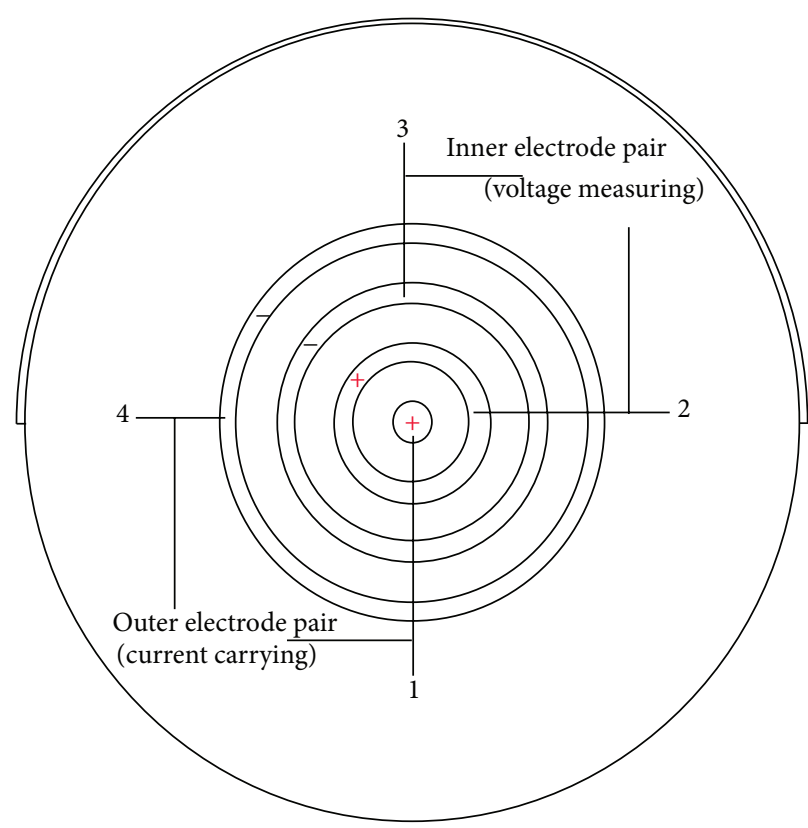

(b)

Figure 1: Arrangement for electrodes of (a) tetrapolar horizontal or vertical rectangle electrode system and (b) tetrapolar horizontal ring electrode system.

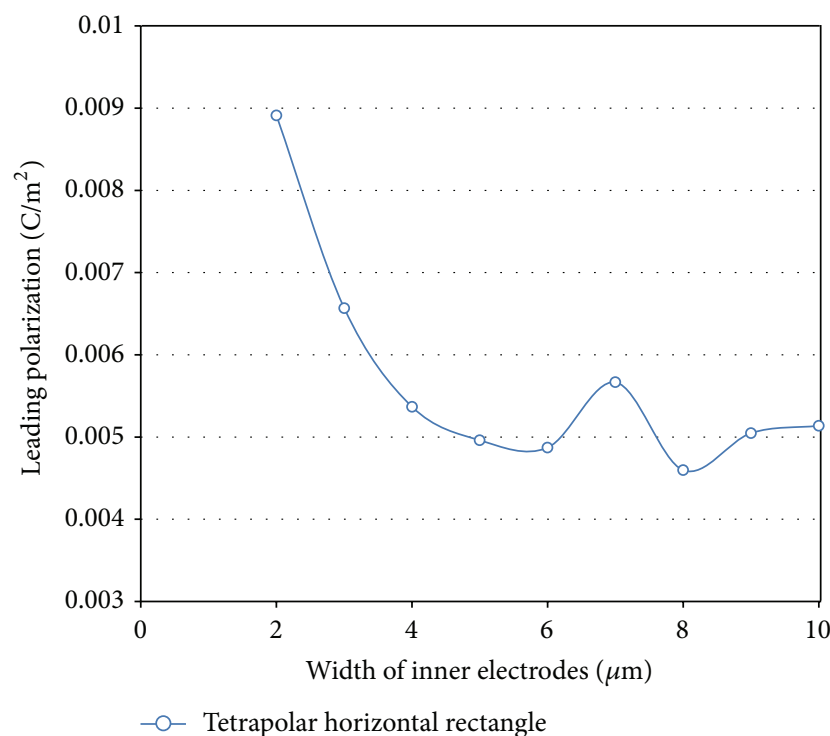

(a)

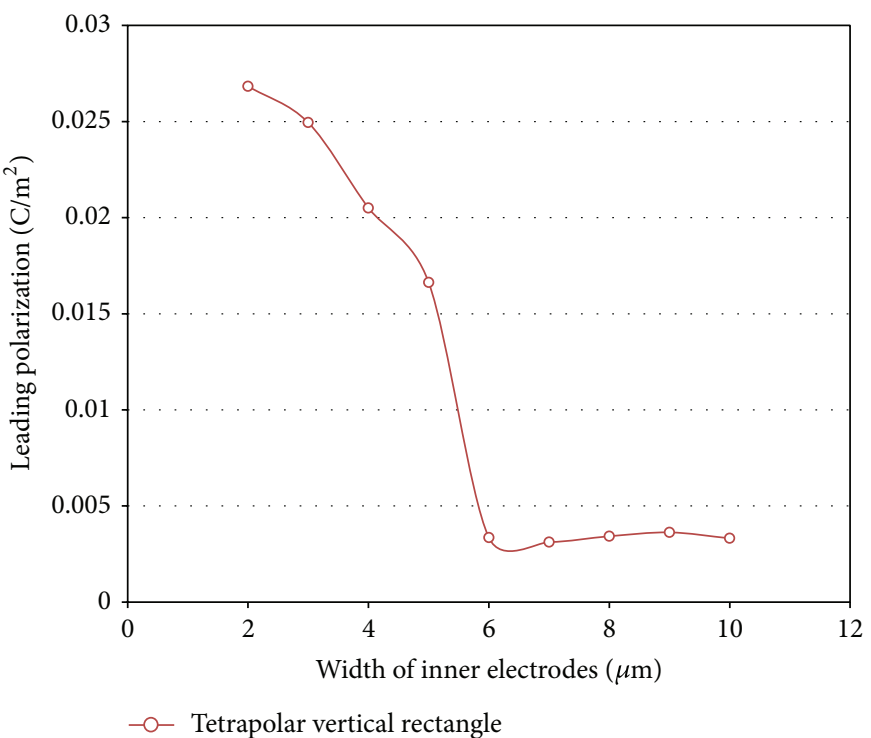

(b)

FIGURE 2: The response of leading polarization pertinent to (a) tetrapolar horizontal rectangle electrode system and (b) tetrapolar vertical rectangle electrode system to the change of width of inner electrode pair (width of outer electrodes: $5 \mu$ m; thickness of electrodes: $100 \mathrm{~nm}$; length of electrodes: $20 \mu \mathrm{m}$; distance between electrodes: $10 \mu \mathrm{m}$ ).

hand, although horizontal ring tetrapolar electrode system has more zones of negative sensitivity than the other two kinds, its degree of uniformity is the highest among them.

\section{Comparison and Analysis}

In order to find out the best setup of electrode system, it is essential to make comparison on polarization and sensitivity between diversity types of electrode system. It can be seen explicitly from what has been mentioned above that the employment of tetrepolar electrode system instead of dipolar electrode system can relieve the phenomenon of polarization to a large extent, which means if the device mainly focuses on lowering the degree of polarization, four-electrode system will be the better alternative. Unfortunately, two-electrode electrode system does have its advantage in sensitivity. There are no zones of negative sensitivity for dipolar electrode 


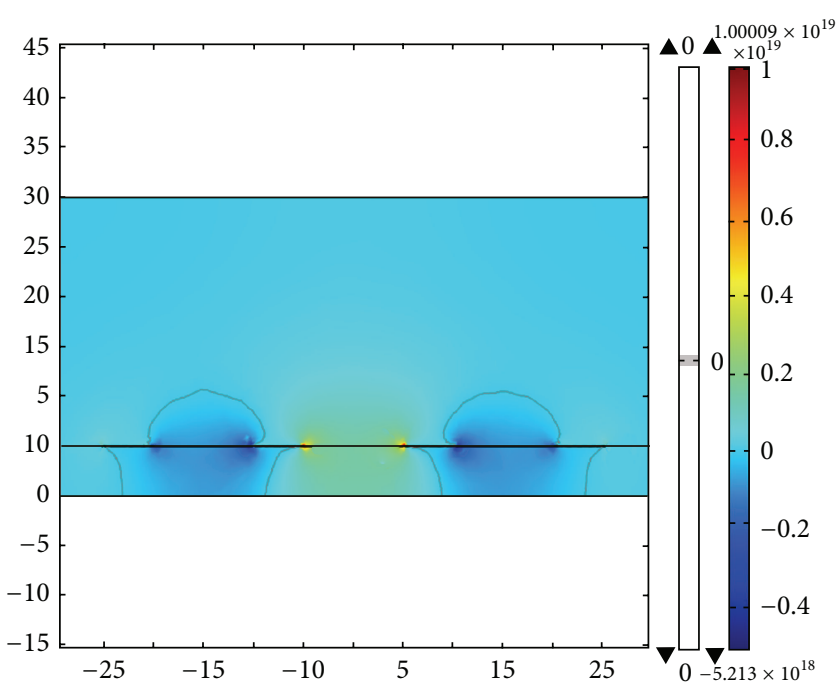

(a)

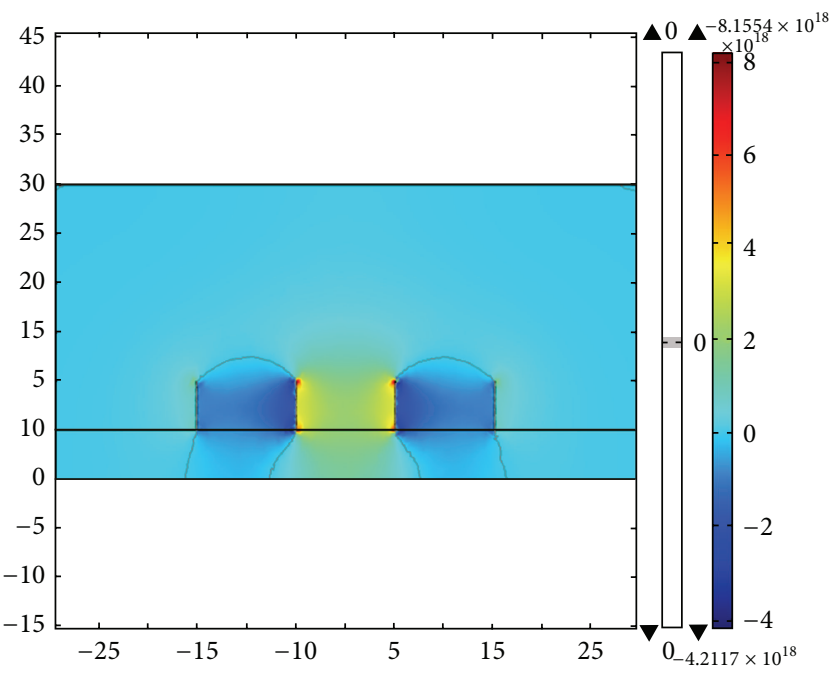

(b)

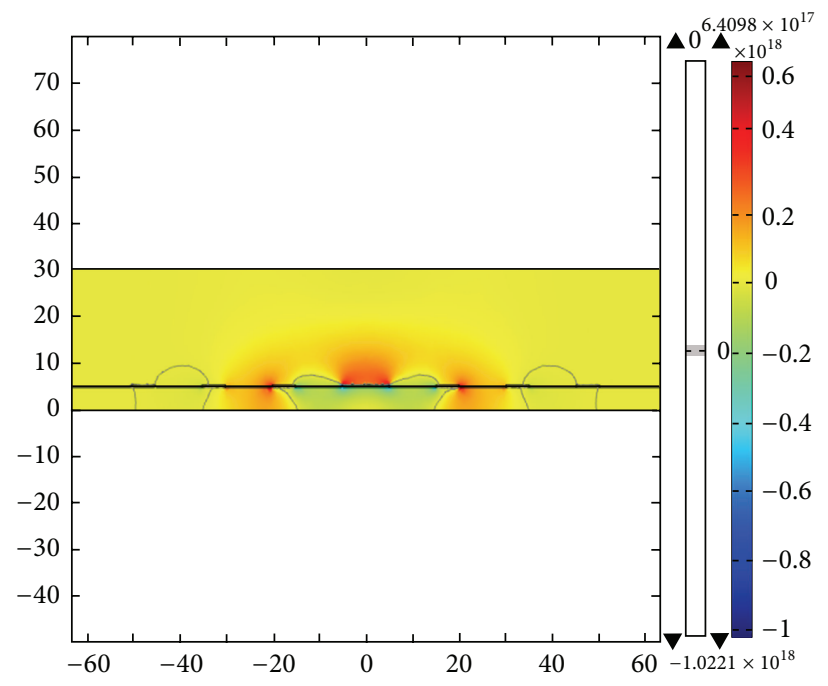

(c)

Figure 3: Sensitivity distribution of (a) tetrapolar horizontal rectangle electrode system, (b) tetrapolar vertical rectangle electrode system, and (c) tetrapolar horizontal ring electrode system.

system in that its electrodes function as current carrying electrodes and voltage measuring electrodes at the same time, which makes the product of $J_{1}$ and $J_{2}$ always positive. As for the factor of geometry, it can be seen clearly from Figure 5 that vertical rectangle electrode system has the highest degree of polarization at the case of two-electrode systems and four-electrode systems. For dipolar electrode system, the degree of polarization pertaining to horizontal ring electrode system is second to that of vertical rectangle electrode system while horizontal rectangle electrode system has the weakest electrode polarization. But the polarization degree in the case of horizontal rectangle and horizontal ring electrode system keeps at the same level. Diversity systems respond differently to the change of dimension and the simulation results relevant to polarization are listed in Table 1 . As a result, horizontal rectangle electrode system is much likely to be the best option for two-electrode system. It is substantial to take the factor of sensitivity when trying to work out the best setup of electrode system when it turns to fourelectrode systems. Meanwhile, the electrode of polarization can be relieved by increasing the distance between electrodes, which will optimize its performance to some extent. From what has been mentioned previously, tetrapolar horizontal rectangle electrode system has the smallest area of negativesensitivity zones although its uniformity of sensitivity is not as good as tetrapolar horizontal ring electrode system. That is to say, horizontal rectangle electrode system may be the best option, of which performance can be improved by increasing the width of inner electrode pair and reducing the distance between current carrying electrode and voltage measuring electrode. 


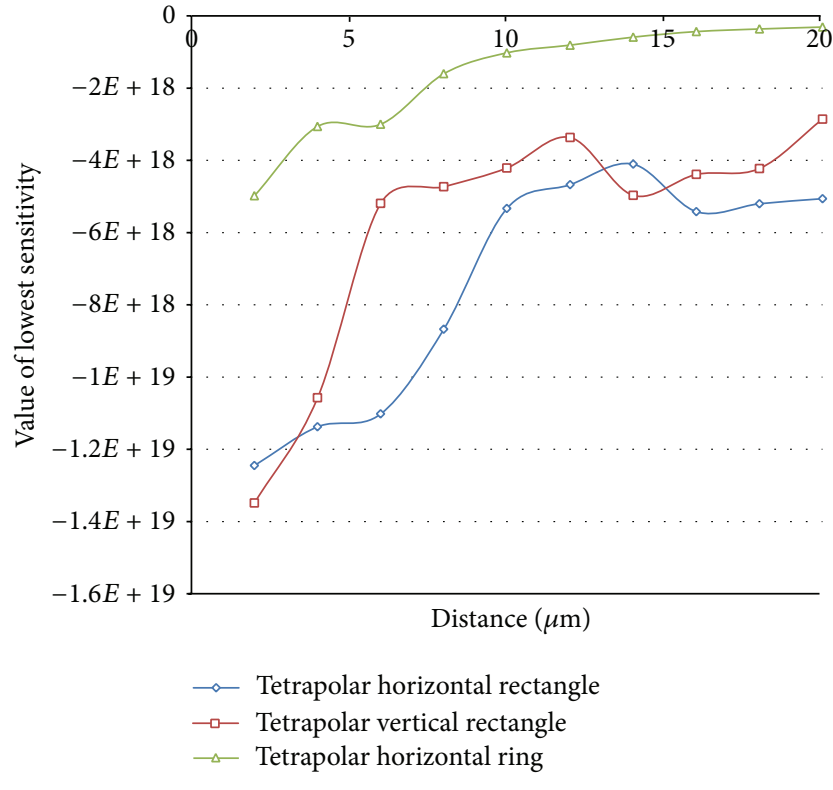

(a)

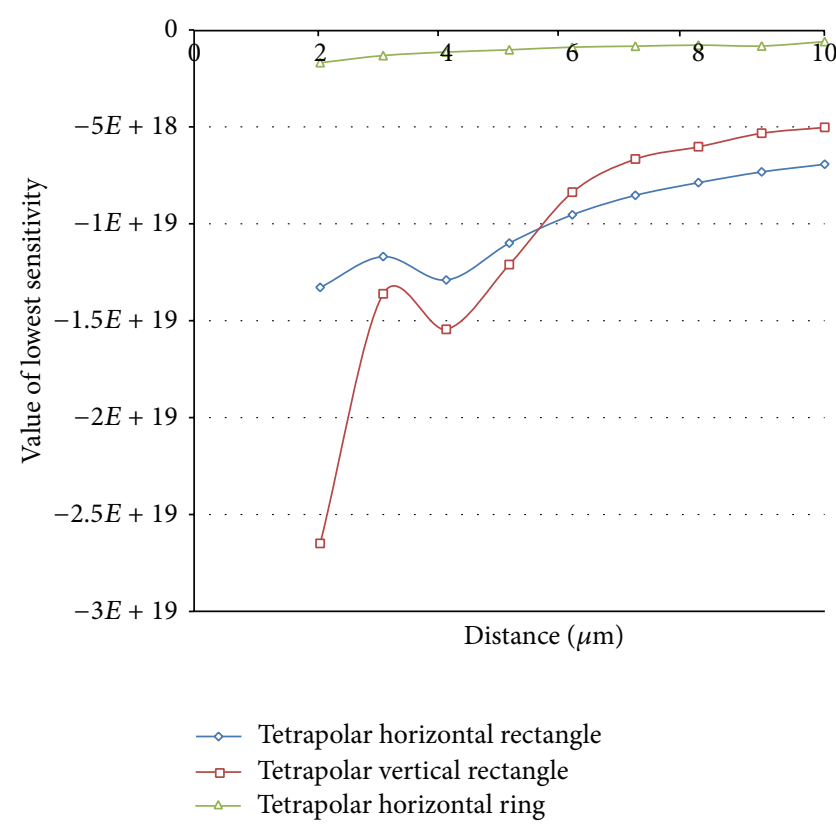

(b)

FIGURE 4: The response of lowest sensitivity pertinent to tetrapolar electrode system to the change of (a) distance between electrodes (width of electrodes: $5 \mu \mathrm{m}$; length of electrodes: $20 \mu \mathrm{m}$; thickness of electrodes: $100 \mathrm{~nm}$ ) and (b) width of inner electrodes (width of outer electrodes: $5 \mu \mathrm{m}$; thickness of electrodes: $100 \mathrm{~nm}$; length of electrodes: $20 \mu \mathrm{m}$; distance between electrodes: $10 \mu \mathrm{m}$ ).

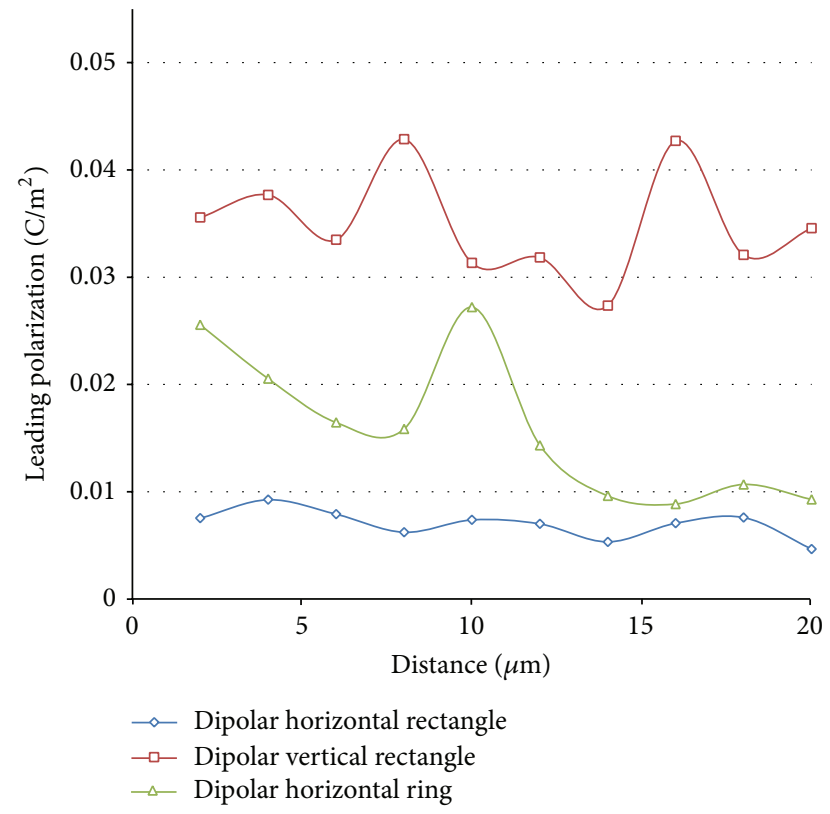

(a)

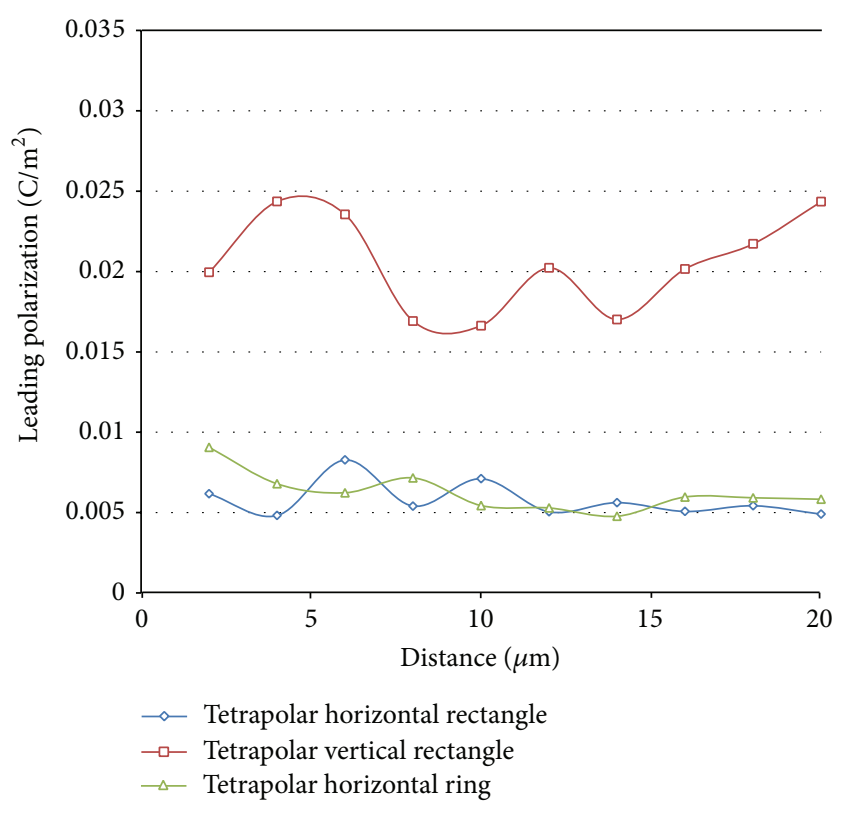

(b)

FIGURE 5: The response of leading polarization pertinent to (a) dipolar and (b) tetrapolar electrode system to the change of distance between electrodes (width of electrodes: $5 \mu \mathrm{m}$; length of electrodes: $20 \mu \mathrm{m}$; thickness of electrodes: $100 \mathrm{~nm}$ ).

\section{Conclusion}

(1) Terapolar electrodes system has less polarization than dipolar electrodes system.

(2) For tetrapolar electrodes system, zones of negative sensitivity exist between current carrying electrodes and voltage measuring electrodes.
(3) For tetrapolar vertical electrodes system, the degree of leading polarization drops remarkably when the width of inner electrodes is larger than the outer ones.

(4) With respect to the polarization of dipolar electrodes system, horizontal rectangle system has the lowest degree. 
TABLE 1: Relief of polarization due to cncreasing parameters.

\begin{tabular}{lccccc}
\hline $\begin{array}{l}\text { Increasing } \\
\text { parameters }\end{array}$ & $\begin{array}{c}\text { Dipolar } \\
\text { horizontal } \\
\text { rectangle }\end{array}$ & $\begin{array}{c}\text { Dipolar vertical } \\
\text { rectangle }\end{array}$ & $\begin{array}{c}\text { Dipolar } \\
\text { horizontal ring }\end{array}$ & $\begin{array}{c}\text { Tetrapolar } \\
\text { horizontal } \\
\text { rectangle }\end{array}$ & $\begin{array}{c}\text { Tetrapolar } \\
\text { vertical rectangle }\end{array}$ \\
\hline $\begin{array}{l}\text { Distance } \\
\text { Thickness }\end{array}$ & $\sqrt{ }$ & $\sqrt{ }$ & & & $\sqrt{ }$ \\
$\begin{array}{l}\text { Width of inner } \\
\text { electrodes }\end{array}$ & - & - & - & $\sqrt{ }$ & $\sqrt{ }$ \\
\hline
\end{tabular}

(5) As to the polarization of tetrapolar electrodes system, horizontal electrodes systems are better than vertical one.

(6) For tetrapolar electrodes system, the degree of negative-sensitivity zones regarding horizontal ring electrodes system is the lowest among the three setups.

(7) By increasing the corresponding parameters listed in Table 1, the performance of the biosensor can be improved to some extent.

\section{Conflict of Interests}

The author declares that there is no conflict of interests regarding the publication of this paper.

\section{References}

[1] H. Mazhab-Jafari, L. Soleymani, and R. Genov, "16-channel CMOS impedance spectroscopy DNA analyzer with dual-slope multiplying ADCs," IEEE Transactions on Biomedical Circuits and Systems, vol. 6, no. 5, pp. 468-478, 2012.

[2] D. M. Garner, H. Bai, P. Georgiou et al., "A multichannel DNA SoC for rapid point-of-care gene detection," in Proceedings of the IEEE International Solid-State Circuits Conference (ISSCC '10), pp. 492-493, February 2010.

[3] J. M. Rothberg, W. Hinz, T. M. Rearick et al., "An integrated semiconductor device enabling non-optical genome sequencing," Nature, vol. 475, no. 7356, pp. 348-352, 2011.

[4] M. Schindler, S. K. Kim, C. S. Hwang, C. Schindler, A. Offenhäusser, and S. Ingebrandt, "Novel post-process for the passivation of a CMOS biosensor," Physica Status Solidi-Rapid Research Letters, vol. 2, no. 1, pp. 4-6, 2008.

[5] D. Gonçalves, D. M. F. Prazeres, V. Chu, and J. P. Conde, "Detection of DNA and proteins using amorphous silicon ion-sensitive thin-film field effect transistors," Biosensors and Bioelectronics, vol. 24, no. 4, pp. 545-551, 2008.

[6] F. Lisdat and D. Schäfer, "The use of electrochemical impedance spectroscopy for biosensing," Analytical and Bioanalytical Chemistry, vol. 391, no. 5, pp. 1555-1567, 2008.

[7] L. Añorga, A. Rebollo, J. Herrán, S. Arana, E. Bandrés, and J. García-Foncillas, "Development of a DNA microelectrochemical biosensor for CEACAM5 detection," IEEE Sensors Journal, vol. 10, no. 8, pp. 1368-1374, 2010.

[8] H. Liu, R. Malhotra, M. W. Peczuh, and J. F. Rusling, "Electrochemical immunosensors for antibodies to peanut allergen Ara h2 using gold nanoparticle-peptide films," Analytical Chemistry, vol. 82, no. 13, pp. 5865-5871, 2010.

[9] O. Pänke, W. Weigel, S. Schmidt, A. Steude, and A. A. Robitzki, "A cell-based impedance assay for monitoring transient receptor potential (TRP) ion channel activity," Biosensors and Bioelectronics, vol. 26, no. 5, pp. 2376-2382, 2011.

[10] A. Manickam, C. A. Johnson, S. Kavusi, and A. Hassibi, "Interface design for CMOS-integrated electrochemical impedance spectroscopy (EIS) biosensors," Sensors, vol. 12, no. 11, pp. 14446-14488, 2012.

[11] S. Grimnes and Ø. G. Martinsen, "Sources of error in tetrapolar impedance measurements on biomaterials and other ionic conductors," Journal of Physics D: Applied Physics, vol. 40, no. 1, pp. 9-14, 2007.

[12] COMSOL, "Product Suite[DB/OL]," 2013, http://www.comsol .com/products.

[13] B.V. Metrohm Autolab, "Fuel cells part 3-characterization using EIS," Autolab Application Note FC03, http://www.ecochemie.nl/download/Applicationnotes/Autolab_Application_Note_FC03.pdf.

[14] S. Grimnes and Ø. G. Martinsen, "Positive phase bioimpedance and system inductive properties," in Proceedings of the World Congress in Medical Physics and Biomedical Engineering, Sydney, Australia, 2003. 


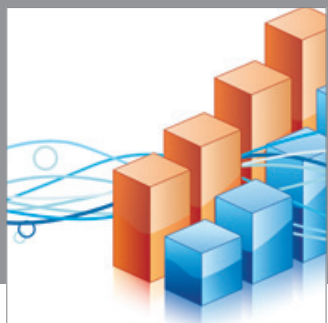

Advances in

Operations Research

mansans

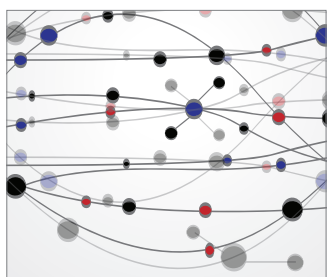

The Scientific World Journal
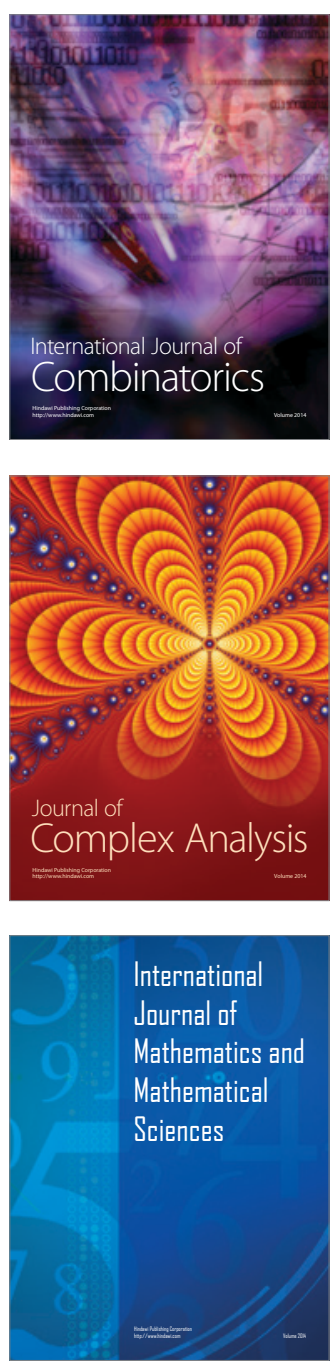
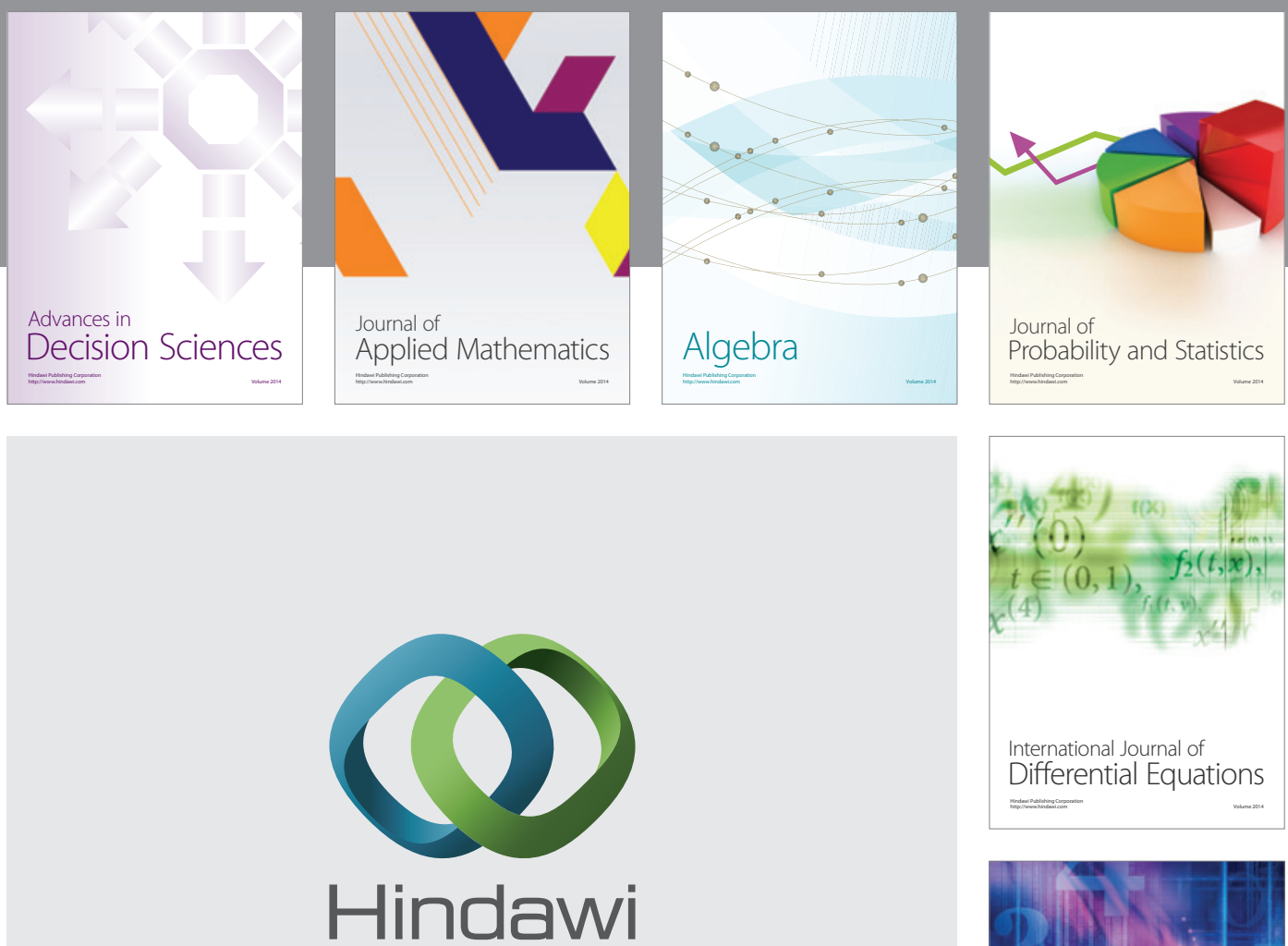

Submit your manuscripts at http://www.hindawi.com
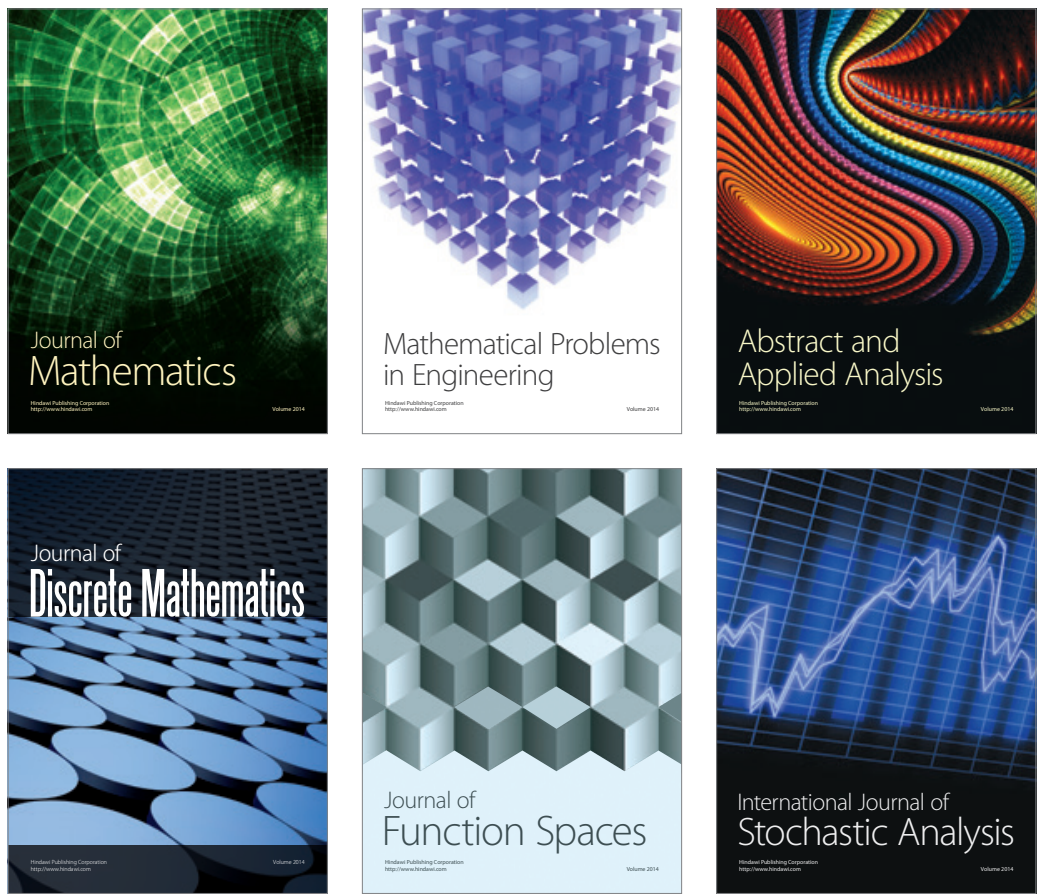

Journal of

Function Spaces

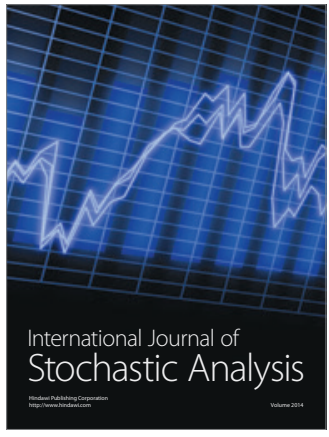

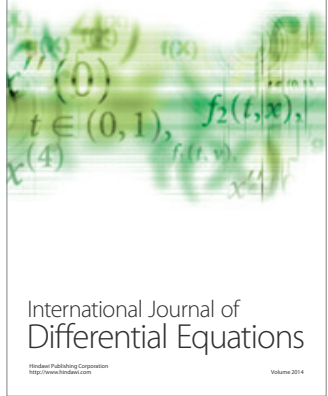
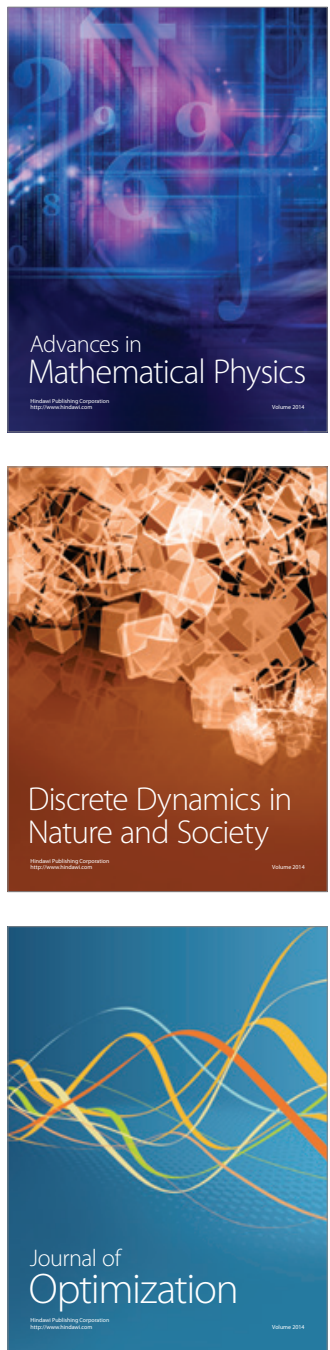